\title{
UN ANÁLISIS COMPARATIVO DE LA INSERCIÓN DE INMIGRANTES LATINOAMERICANOS EN EL MERCADO DE TRABAJO DE ESPAÑA EN 2001 ${ }^{1}$
}

\author{
A COMPARATIVE ANALYSIS OF THE INSERTION OF LATIN AMERICAN IMMIGRANTS \\ IN THE SPANISH LABOR MARKET IN 2001
}

\author{
Elaine Meire Vilela ${ }^{2}$ \\ Cláudia Lima Ayer de Noronha ${ }^{3}$ \\ Universidade Federal de Minas Gerais, Minas Gerais, Brasil
}

\begin{abstract}
Resumen: Este trabajo tiene el objetivo de realizar un análisis comparativo de la situación socioeconómica de los inmigrantes latinoamericanos de origen ecuatoriano, colombiano, boliviano, peruano, venezolano y brasileño, incorporados al mercado de trabajo en España, en 2001. Se pretende investigar si existen diferencias niveles de empleo, comparando los seis grupos de latinoamericanos, y cuáles están en mejores o peores ocupaciones en el mercado de trabajo de España. También tenemos como objetivo identificar si esas diferencias de inserción laboral pueden ser explicadas por la nacionalidad de esos trabajadores, controlando las características individuales de esos trabajadores. Para investigar estos puntos, seleccionamos a los extranjeros ecuatorianos, colombianos, bolivianos, peruanos, venezolanos y brasileños, entre 25 y 65 años, en el Censo Demográfico español. En cuanto a los modelos de análisis, estimamos dos modelos de regresión logística, para verificar las diferencias relacionadas al origen étnica/nacional para la determinación de la empleabilidad e inserción ocupacional. Se mantienen las características socioeconómicas de los trabajadores constantes (sexo, edad, escolaridad, entre otras características), verificar el efecto del país de nacimiento del trabajador para explicar la situación del inmigrante en el mercado de trabajo español.
\end{abstract}

Palabras clave: Inmigrantes latinoamericanos, desigualdad social, mercado de trabajo español, inserción socio-ocupacional.

Abstract: The purpose of this study is to conduct a comparative analysis of the socioeconomic situation of Latin American immigrants from Ecuador, Colombia, Bolivia, Peru, Venezuela and Brazil, who were incorporated into the labor market in Spain in 2001. The aim is to investigate whether there are differences in levels of employment, comparing the six Latin American groups, and which are in better or worse occupations in the Spanish labor market. We also intend to identify if these differences in labor insertion can be explained by the nationality of these workers, controlling the individual characteristics of these workers. To investigate these points, we selected the Ecuadorian, Colombian, Bolivian, Peruvian, Venezuelan and Brazilian foreigners, between 25 and 65 years old, in the Spanish Demographic Census. Regarding the

\footnotetext{
${ }^{1}$ Destacamos aquí el agradecimiento a Integrated Public Use Microdata Series - IPUMS por el acceso a los micro datos del censo demográfico de España.

${ }^{2}$ Professor associado do Departamento de Sociologia da Universidade Federal de Minas Gerais. Doutorado em Ciências Humanas (Sociologia e Ciência Política) da Universidade Federal de Minas Gerais (2008). Email: elainevilela@fafich.ufmg.br.

${ }^{3}$ Ph.D. em Ciências Humanas (Sociologia) da Universidade Federal de Minas Gerais (2018). Departamento de Sociologia da Universidade Federal de Minas Gerais. Email: claudiaayer@gmail.com.
} 
analysis models, we estimated two models of logistic regression, to verify differences related to ethnic / national origin for the determination of employability and occupational insertion. If we keep the socioeconomic characteristics of the workers constant (gender, age, schooling, among other characteristics), we will verify the effect of the country of birth of the worker to explain the situation of the immigrant in the Spanish labor market.

Keywords: Latin American immigrants, social inequality, Spanish labor market, sociooccupational insertion. 


\section{INTRODUCCIÓN}

Este trabajo tiene como objetivo central realizar un análisis comparativo de la situación socioeconómica de inmigrantes latinoamericanos de origen ecuatoriana, colombiana, boliviana, peruana, venezolana y brasileña, insertos en el mercado de trabajo en España en el año 2011. Se pretende investigar si hay diferencias en la inserción laboral de estos grupos de extranjeros en el mercado español. Entre esos grupos de latinoamericanos, ¿existen diferencias en los niveles de empleo y desempleo en el mercado de trabajo español? Para aquellos que se encuentran insertos en el mercado de trabajo, ¿qué grupos están en mejores situaciones ocupacionales? Si se verifican diferencias entre la empleabilidad e inserción socio-ocupacional, ¿el origen étnico/nacional del trabajador es un factor de explicación de tales desigualdades? Es decir, mantenidas las características socioeconómicas de los trabajadores constantes (sexo, edad, escolaridad, entre otras), analizaremos si el país de nacimiento del trabajador es una variable explicativa de la situación del inmigrante en el mercado de trabajo español.

La relevancia de este tema de estudio reside en dos factores. El primero se halla en el hecho de que, a principios del siglo XXI, España vivió una fase de entrada creciente de trabajadores de origen latinoamericano (GIL ARAUJO, 2005). Se observa un aumento en el número de inmigrantes de 61.000 en 1991 a 514.485 a finales de 2003 (EXTRANJERÍA, 2004), representando el 31,2\% de la inmigración regularizada en el país. Este fenómeno de evolución de inmigrantes latinoamericanos es, en parte, resultado de acuerdos firmados por España con Ecuador y Colombia para incentivar la contratación de trabajadores provenientes de estos países (ESCRIBANO, DE LERA Y BUJÁN, 2002). Estas políticas e estímulos tuvieron impactos inmediatos sobre las estadísticas de inmigrantes, situando a los ecuatorianos como la segunda mayor población extranjera documentada en España, seguidos por los colombianos y superados apenas por los marroquíes (GIL ARAUJO, 2005).

El segundo factor se refiere al discurso homogeneizador de la inserción de los inmigrantes en el mercado de trabajo español. Un grupo importante de trabajos sobre el tema sostiene que estos inmigrantes se introducen en los estratos más bajos y desvalorizados de la estructura ocupacional del mercado laboral español (RIPOLL, 2008). Sin embargo, se sabe, a partir de estudios anteriores (VAN TUBERGEN, MAAS Y FLAP, 2004; FERNÁNDEZ Y ORTEGA, 2008; VILELA, 2011; VILELA, COLLARES Y NORONHA, 2015, entre otros) 
que el origen es un factor que influye en la situación del inmigrante en el mercado de trabajo dando lugar a una heterogeneidad étnica/nacional con respecto a la ubicación en la jerarquía socio-ocupacional establecida en el mercado de trabajo. Es decir, cuando se analizan diversos grupos de inmigrantes de diferentes países de origen con un destino similar, se observa que, tras controladas las características de los individuos y del lugar de destino, así como la situación del mercado, el país de origen mantiene alta correlación con la localización del individuo en la estructura jerárquica socioeconómica. Se observa que algunos grupos de inmigrantes se encuentran en ventaja y otros en desventaja, dependiendo del grupo de origen de referencia (JONG Y MADAMBA, 2001; VAN TUBERGEN, MAAS Y FLAP, 2004; VILELA, 2011; VILELA, COLLARES Y AYER DE NORONHA, 2012).

A pesar de ello, no hay estudios que midan comparativamente si en el mercado de trabajo español hay diferencias de inserción laboral entre inmigrantes latinoamericanos que se explican por el país de nacimiento del extranjero, incluso después del control de las características individuales de los ciudadanos trabajadores internacionales. De esta forma, creemos que este trabajo es importante porque pretende sanar tales lagunas en la bibliografía existente sobre el asunto en España.

Para responder a las preguntas planteadas, se ha utilizado un enfoque cuantitativo, mediante la aplicación de modelos estadísticos a partir de datos del Censo Demográfico de España de 2001. A partir del este Censo, seleccionamos a todos los extranjeros hombres ${ }^{4}$ de origen ecuatoriano, colombiano, boliviano, peruano, venezolano y brasileño, con edades comprendidas entre los 25 y los 65 años $^{5}$. En relación con los modelos de análisis, calculamos dos modelos de regresión logística para verificar las diferencias relativas al origen étnico/nacional para la determinación de la empleabilidad y la inserción laboral.

4 Destacamos que el análisis será realizado apenas para hombres porque: 1) existen evidencias de una segregación ocupacional por sexo clara en el mercado de trabajo en el país (Ripoll, 2008; Fernández y Ortega, 2008); 2) hay diferencias proporcionales significativas en la representatividad de hombres y mujeres entre esos inmigrantes en España. Por ejemplo, en 2005, entre los latinoamericanos el porcentaje es de 54\% de mujeres y $46 \%$ de hombres. Ya entre los brasileños, específicamente, el porcentaje es de $64 \%$ de mujeres y $36 \%$ de hombres (Ripoll, 2008); 3) ya hay muchos grupos que están siendo comparados y llevar a cabo un análisis para hombres y mujeres separadamente podría dejar la lectura del texto ardua y desgastante. Destacamos que es de nuestro interés, después de este estudio, realizar un análisis semejante para la inserción de las mujeres en el mercado de trabajo español.

5 Este rango de edad se justifica por la idea de la entrada en el mercado del trabajo tras la probable finalización de estudios superiores (25 años); por la edad de jubilación (65 años); por la mayor representación del inmigrante trabajador en la edad joven adulta (rango de 25 a 44 años de edad). 
Para presentar el estudio de la mejor forma posible, dividimos el texto en tres apartados, además de esta introducción y de las consideraciones finales. En el primer apartado, presentamos una revisión de la bibliografía que trata las formas de inserción de los inmigrantes en el mercado de trabajo para introducir sus determinantes potenciales. Con base en esta discusión, elaboramos las hipótesis que contrastaremos en los apartados siguientes. En la segunda parte, abordamos la metodología utilizada para probar nuestras suposiciones. En este momento presentamos los datos y los modelos estadísticos usados, explicando la construcción de las variables introducidas en los modelos, la cual se fundamenta en el debate teórico anterior. Por último, en el tercer apartado, exponemos el análisis de los datos. Introducimos de forma sucinta un análisis descriptivo y en seguida el análisis de los modelos demostrando la validez o no de las hipótesis establecidas a priori. 


\section{REVISIÓN DE LA BIBLIOGRAFÍA}

El tema de la inmigración se convirtió en un asunto bastante estudiado y discutido (por los investigadores y gestores públicos e, incluso, por la población en general) una vez que, a partir de los años 1980, España pasó de ser un país de emigración a ser receptor de inmigrantes. Los principales tópicos de interés de los investigadores sobre el asunto se refieren a la política migratoria, a los determinantes de la migración, a la asimilación del inmigrante en la sociedad y a la inserción del inmigrante en el mercado de trabajo (ver, por ejemplo, AMUEDODORANTES Y DE LA RICA, 2006; FERNÁNDEZ Y ORTEGA, 2008; RIPOLL, 2008; IZQUIERDO, LACUESTA Y VEGAS, 2009; SANROMÁ, RAMOS Y SIMÓN, 2009; ARTILES, LÓPEZ-ROLDÁN Y MOLINA, 2011).

Con respecto a este último tópico, que es del interés de este artículo, hay diversos estudios que tratan de explicar la situación del inmigrante en el mercado de trabajo (AMUEDODORANTES Y DE LA RICA, 2006; FERNÁNDEZ Y ORTEGA, 2008; IZQUIERDO, LACUESTA Y VEGAS, 2009).

En general, estos estudiosos identificaron que los inmigrantes, cuando acceden al mercado de trabajo español, presentan tasas de empleo similares a las de los nativos, pero rentas y estatus socioeconómico ocupacional inferiores. Este grupo se concentra en las ocupaciones del mercado secundario, que son inestables, mal remuneradas, de contratos temporales, y de baja cualificación, comparadas con las del mercado primario ${ }^{6}$. Sin embargo, tras cinco años de estancia en la sociedad de acogida (España), los inmigrantes tienden a superar las tasas de empleo frente a los nativos e incluso alcanzan ocupaciones y rentas similares o inclusive superiores a las de los nativos (AMUEDO-DORANTES Y DE LA RICA, 2006; FERNÁNDEZ YORTEGA, 2008; RODRÍGUEZ-PLANAS Y NOLLENBERGER, 2014).

Los investigadores muestran que existe una movilidad social, aunque pequeña, para los inmigrantes (AMUEDO-DORANTES Y DE LA RICA, 2006; FERNÁNDEZ Y ORTEGA, 2008). Por ejemplo, a partir de los datos del Labour Fource Survey de 1996 a 2005, Fernández y Ortega (2008) identifican que, tras cinco años de residencia en España, la diferencia con respecto a la participación en la fuerza de trabajo entre nativos e inmigrantes disminuye y 
mejora la situación de los inmigrantes; las tasas de desempleo se reducen para los inmigrantes y llegan a ser menores que las de los nativos. No obstante, los contratos temporales y la sobreeducación se mantienen con tasas significativas para los inmigrantes después de cinco años de permanencia en la sociedad de acogida. Parte de la explicación de tal mejoría en la situación socioeconómica del inmigrante en el mercado de trabajo se encuentra en la teoría de la asimilación. Según esta teoría, después de algún tiempo en la sociedad de acogida, la situación de los inmigrantes tiende a mejorar, llegando a un nivel semejante o incluso superior al de los nativos ${ }^{7}$.

Cabe destacar que estos estudios revelan que el origen del inmigrante es un factor que influye en la situación del mismo en el mercado de trabajo. Fernández y Ortega (2008) muestran que los resultados sobre la situación en el mercado laboral son muy distintos si se consideran el género y el origen. Con relación al origen, los autores observan que, entre inmigrantes del Este europeo, de África y de América Latina, existen grandes diferencias en cuanto a la situación socioeconómica ocupacional. Para el análisis los autores estiman ecuaciones de regresión lineales, en las cuales las variables respuestas son: situación de desempleo, participación en la fuerza de trabajo e inserción en el mercado de trabajo a través de contratos temporales. Las variables explicativas son el número de años que el extranjero vive en España, edad, años de escolaridad, identificación del trabajador es nativo o extranjero y, para los inmigrantes, el origen del trabajador. Los resultados de la investigación demuestran que los extranjeros del Este europeo se encuentran en mejor situación en el mercado de trabajo (a veces incluso comparados a los nativos) que latinoamericanos y africanos. Estos últimos son los que están en peor situación, en cuanto a la tasa de desempleo, participación en la fuerza de trabajo y contratos temporales de trabajo.

De forma semejante a las observaciones de Fernández y Ortega (2008), AmuedoDorantes y De la Rica (2006) constatan que la asimilación ocupacional y de empleo varía por sexo, origen y nivel educativo. En su estudio, se entiende que los inmigrantes originarios de la Unión Europea no se diferencian de los nativos en cuanto a la probabilidad de estar o no empleados y con respecto al estatus socioeconómico ocupacional. Para la realización del estudio, utilizan datos del Censo Demográfico español de 2001 y aplican al banco de datos modelos econométricos, cuya variable respuesta son la situación de empleo o desempleo, el 
rendimiento y el status ocupacional. En cambio, los demás inmigrantes (esto es, otros europeos, africanos y latinoamericanos) se encuentran en peor situación. Entre estos últimos, los europeos no comunitarios y los latinoamericanos alcanzan mejores niveles de empleo y ocupación con el tiempo, mientras que existe muy poca evidencia de que esta situación ocurra con los extranjeros de origen africano.

Rodríguez-Planas y Nollemberger (2014) refuerzan estos hallazgos. Ellos demuestran que cuando llegan los inmigrantes se sitúan entre las ocupaciones de la base de la pirámide laboral (de baja cualificación). Pero pasados cinco años de estancia en la sociedad de destino, se desplazan hacia puestos mejores de cualificación media. Además de esto, los autores llaman la atención sobre el efecto que tiene el origen. Destacan que la situación de los inmigrantes provenientes de otros países miembros de la Unión Europea (UE) es buena incluso en el momento de llegada a España. Esto es, los extranjeros de esta región son menos propensos a ser empleados en trabajos poco cualificados. Están en empleos de la cima de la jerarquía ocupacional (incluso comparados con los nativos). Los otros grupos están sobrerrepresentados en trabajos de baja calificación (a pesar de que esa sobrerrepresentación se reduzca con el paso del tiempo). Latinoamericanos e inmigrantes del Este europeo tienen más probabilidad de acceder a puestos de cualificación media que los africanos.

A partir de estos resultados formulamos las siguientes cuestiones: ya que el origen es un factor que influye en la situación del inmigrante en el mercado de trabajo en la sociedad de acogida, ¿quién está en peor o mejor situación en el mercado de trabajo dentro del grupo de los latinoamericanos, considerando los niveles de empleo y la inserción ocupacional de los trabajadores? ¿Son brasileños, ecuatorianos, colombianos, bolivianos, peruanos o venezolanos?

Como podemos observar a partir de la bibliografía sobre el tema, la región de origen del inmigrante ha sido el centro de los estudios sobre la situación del inmigrante en el mercado de trabajo español. Sin embargo, los estudios, en general, agregan individuos de diferentes países, construyendo una categoría única, como por ejemplo los "latinoamericanos". De esta forma, tal estrategia metodológica resulta problemática, dado que entre esos países existen diferencias socioeconómicas y culturales que influyen en las formas de inserción y alcance ocupacional de sus miembros en el mercado de trabajo (WILSON Y PORTES, 1980; VAN TUBERGEN, MAAS Y FLAP, 2004; PORTES Y SHAFER, 2007).

A partir de esta discusión sobre homogeneidad y/o heterogeneidad existente entre diversos países latinoamericanos, entendido como los diferentes símbolos, hábitos y demás 
aspectos específicos de cada país cultural, surge la idea de considerar la distancia social que los nativos sienten en relación con los inmigrantes (PORTES Y RUMBAUT, 2001), entendida como el grado de "proximidad" entre personas o grupos (VAN TUBERGEN, MAAS Y FLAP, 2004). De acuerdo con Portes y Rumbaut (2001), el grado de distancia social es mayor cuanto más diferente sea la cultura, la apariencia física y el background socioeconómico entre nativos e inmigrantes. Varios investigadores sostienen que la distancia social entre nativos y grupos de inmigrantes da lugar a discriminación en el mercado de trabajo (PORTES Y RUMBAUT, 2001; VAN TUBENGER, MAAS Y FLAP, 2004).

Con base en dicha teoría, establecimos una hipótesis que será verificada en este estudio, a saber: 1) con base en la teoría de la distancia social, suponemos que los brasileños (por el hecho de que Brasil fue colonizado por Portugal y no por España y debido a que el idioma oficial es el portugués y no el español) mantienen una distancia social mayor con los nativos (comparados con otros extranjeros latinoamericanos). Por tanto, tienden a estar en peor situación en el mercado de trabajo que otros inmigrantes provenientes también de América Latina.

Otro punto a considerar, según Tienda y Lii (1987) y Van Tubergen, Maas y Flap (2004) es el tamaño relativo del grupo inmigrante, el cual influye en la situación económica del individuo. De acuerdo con Tenda y Lii (1987), los prejuicios y la discriminación aumentan con el tamaño del grupo inmigrante, ya que grupos grandes son más visibles y entonces percibidos por la población nativa como amenaza potencial en términos de poder económico y político. De esta forma, la sociedad de acogida trataría de imponer penalizaciones a miembros de tales grupos. Por eso, la hipótesis es la de que, cuanto mayor es el grupo étnico, menor es la realización del mismo en el mercado de trabajo. Por otro lado, otros investigadores han sugerido que grupos grandes de inmigrantes tienen mejor desempeño en el mercado de trabajo (PORTES Y BACH, 1985; PORTES Y RUMBAUT, 2001; VAN TUBERGEN, MAAS Y FLAP, 2004) debido a su "capital étnico".

El capital étnico (medido muchas veces por las redes sociales, economía étnica y enclave étnico) puede proporcionar a los inmigrantes empleos que no son accesibles en el mercado de trabajo común o puede facilitar la iniciativa empresarial o el empleo autónomo en nichos o enclaves étnicos que los nativos no ocupan (PORTES Y BACH, 1985). Se sostiene que este recurso proporciona a los trabajadores inmigrantes rendimientos superiores que a aquellos inseridos en el mercado abierto, en el que hay competencia directa con el nativo. En ese caso 
sería una vía alternativa para la movilidad ascendente de inmigrantes que pueden ser penalizados por barreras lingüísticas y culturales en el mercado de trabajo principal (PORTES Y BACH, 1985; KESLER Y HOUT, 2010).

Sin embargo, tal y como señalado por Portes (2000) y Lin (2006), redes sociales bastante homogéneas son más propensas a presentar desventajas significativas para los inmigrantes, comparadas con las más heterogéneas, dado que pueden cerrar otras oportunidades, tal vez por falta de información que estarían disponibles en el mercado abierto.

Teniendo en cuenta este debate y la idea de amenaza étnica, surge una segunda hipótesis sujeta a verificación: comparados con los brasileños (el grupo menor en números absolutos entre los latinoamericanos) los ecuatorianos (el grupo mayor) se encuentran en peor situación ya que pueden ser vistos como una amenaza étnica.

Destacamos que, manteniendo todo lo demás constante (variables como sexo, edad, nivel educativo, entre otras características) comprobaremos qué tanto y cómo el origen explica la situación del inmigrante en el mercado de trabajo español y pondremos a prueba las dos hipótesis planteadas anteriormente. Para ello, los estudios realizados indican que algunas dimensiones importantes deben ser controladas, y éstas son consideradas en el presente trabajo. Por lo que se refiere a las características individuales del inmigrante, Nee y Sanders (2001) observan que los trabajadores extranjeros llegan con diferentes capitales - humano, financiero y social - que pueden influir en el rendimiento y en la forma de inserción en el mercado de trabajo. Por ejemplo, la acumulación de capital humano ${ }^{8}$, según la bibliografía existente (BECKER, 1962), aumenta la productividad de los trabajadores, contribuyendo para la mejoría del alcance ocupacional ${ }^{9}$. No obstante, Pichler (2011) resalta que inmigrantes internacionales enfrentan una situación diferente de los trabajadores nativos, ya que sus habilidades derivan de una formación extranjera y pueden ser evaluadas con un mayor grado de dificultad por parte de los empleadores. Además, la teoría del capital humano también muestra que la experiencia en

8 Esta teoría parte del principio de que, así como una empresa invierte en capital financiero para garantizar una cierta rentabilidad o beneficio en el mercado, los individuos también invierten en recursos para aumentar su productividad (Becker, 1962). En esa línea, Becker (1962) y Mincer (1958) desarrollaron trabajos de referencia para la teoría del capital humano, concluyendo que las diferencias deremuneración entre los trabajadores tienden a volverse más acentuadas en función de la existencia de niveles diferenciados de experiencia profesional, formación y cualificación.

9 Otros estudiosos afirman que es una visión simplista y argumentan que variables de capital cultural, o sea, de origen social (clase social, etnia) y de significado social (raza, estado civil, etc.) determinan también el alcance ocupacional y la riqueza de los individuos (Collins, 1979; Portes y Bach, 1985; Coleman, 1988; Hasenbalg y Valle Silva, 2003; Aguiar, 2007). 
el mercado de trabajo, que en este estudio tiene como proxy la edad del trabajador, influye en la situación del inmigrante y, consecuentemente, debe ser incorporada en los análisis, tal y como describimos en el próximo apartado de este trabajo.

\section{METODOLOGÍA}

Para la realización de este estudio usamos una submuestra de los datos del Censo Demográfico español de 2001, a partir de la selección de hombres en el mercado laboral, entre 25 y 65 años, empleados y desempleados, de origen ecuatoriano, colombiano, boliviano, peruano, venezolano y brasileño. La muestra utilizada contiene 127 brasileños (2,25\%), 155 venezolanos (2,74\%), 163 bolivianos (2,88\%), 427 peruanos (8,34\%), 1.736 colombianos (30, $69 \%)$ y 3.004 ecuatorianos $(53,1 \%)$, dando un total de 5.657 inmigrantes, muestra estadísticamente representativa del universo. Antes de discutir los modelos estadísticos empleados, se presentan las variables dependientes e independientes (de test y de control) usadas en la investigación.

Tabla 1 - Variables dependientes e independientes

\begin{tabular}{|c|c|c|}
\hline Variable & Tipo & Descripción \\
\hline \multicolumn{3}{|c|}{ Variables dependientes } \\
\hline Empleado & Binaria & $1=$ empleado $/ 0=$ desempleado \\
\hline Trabdesc & & $1=$ Posee trabajo en ocupaciones elementales ${ }^{10}$ \\
\hline $\begin{array}{c}\text { (Trabajo de baja } \\
\text { calificación) }\end{array}$ & Binaria & $0=$ No posee trabajo en ocupaciones elementales \\
\hline \multicolumn{3}{|c|}{ Variables de test } \\
\hline Origen & & \\
\hline Boliviano & Binaria & $1=$ boliviano $/ 0=$ brasileño \\
\hline Colombiano & Binaria & $1=$ colombiano $/ 0=$ brasileño \\
\hline Venezolano & Binaria & $1=$ venezolano $/ 0=$ brasileño \\
\hline
\end{tabular}

10 Se entienden como ocupaciones elementales aquellas definidas por la International Standard Classification of Occupations (ISCO) como "Elementary occupations". 


\begin{tabular}{|c|c|c|}
\hline Peruano & Binaria & $1=$ peruano $/ 0=$ brasileño \\
\hline Ecuatoriano & Binaria & $1=$ ecuatoriano $/ 0=$ brasileño \\
\hline \multicolumn{3}{|c|}{ Variables de control } \\
\hline Edad_cen ${ }^{11}$ & Continua $^{12}$ & Edad del individuo, en años, centralizada ${ }^{13}$ \\
\hline Tiempomigra & Continua & Tiempo de migración en años \\
\hline \multicolumn{3}{|l|}{ Educación } \\
\hline Educ1 & \multirow{4}{*}{$\begin{array}{l}\text { Binaria } \\
\text { Binaria } \\
\text { Binaria } \\
\text { Binaria }\end{array}$} & $1=\sin$ instrucción $/ 0=$ superior completa \\
\hline Educ2 & & $1=$ primaria completa $^{14} / 0=$ superior completa \\
\hline Educ3 & & $1=$ secundaria completa $^{15} / 0=$ superior completa \\
\hline Educ4 & & $1=$ técnica completa $^{16} / 0=$ superior completa \\
\hline Hrtrabs & Discreta & Horas trabajadas por semana \\
\hline \multicolumn{3}{|l|}{ Estado civil } \\
\hline Soltero & Binaria & $1=$ Soltero $/ 0=$ Casado/separado/viudo \\
\hline \multicolumn{3}{|l|}{ Vínculo laboral } \\
\hline Autónomo & Binaria & $1=$ Autónomo $/ 0=$ asalariado \\
\hline Voluntario & Binaria & $1=$ Voluntario $/ 0=$ asalariado \\
\hline \multicolumn{3}{|l|}{ Sector } \\
\hline Rígido $^{17}$ & Binaria & $1={\text { sector rígido } / 0=\text { sector } \text { tradicional }}^{18}$ \\
\hline
\end{tabular}

11 La edad es usada aquí como proxy de experiencia en el mercado de trabajo.

12 Para resolver el problema de la autcorrelación de la variable edad y edad al cuadrado, se llevó a cabo la centralización de esas variables, que consiste en restar el valor de la edad al valor de su media en la muestra analizada (siendo ésta la edad centralizada).

13 Inicialmente, la variable edad era discreta, en un intervalo de 25 a 65, después de la centralización se obtuvo un intervalo continuo de datos de -10.21 a 29,78 años.

14 La categoría "primaria completa" se refiere a "Primary completed".

15 La categoría"secundaria completa" se refiere a la suma de las siguientes: "Lower secondary general completed", "Secondary, general track completed", "Secondary, technical track completed".

16 La categoría "técnica completa" se refiere a "Post-secondary technical education".

17 El sector rígido es la suma de las siguientes categorías: "Manufacturing", "Electricity, gas and water" y "Construction".

18 El sector tradicional es la suma de las siguientes categorías: "Agriculture, fishing, and forestry" y "Mining". 


\begin{tabular}{|l|l|l|}
\hline Flexible $^{19}$ & Binaria & $1=$ sector flexible/ $0=$ sector tradicional \\
\hline Público $^{20}$ & Binaria & $1=$ sector público/ $0=$ sector tradicional \\
\hline
\end{tabular}

Con respecto al método estadístico empleamos la regresión logística binomial con los siguientes objetivos: 1) analizar si el origen étnico influye en la determinación de las probabilidades de que los inmigrantes estén empleados; 2) constatar la realización del estatus ocupacional de los migrantes en el mercado laboral de destino a través de la inserción en puestos de trabajo de baja calificación. Para ello, calculamos dos ecuaciones: en la primera estimación la variable respuesta es binaria, 1 significa que la persona está inserida en el mercado y 0 se refiere a las que están desempleadas. Y para la segunda estimación, 1 significa que la persona está inserida en una ocupación de baja calificación y 0 se refiere a las que no están en esos puestos en el mercado de trabajo. La técnica estadística adoptada, la regresión logística binomial, pemite analizar la variable de prueba (origen ético de los inmigrantes), manteniendo las demás características socioeconómicas de los trabajadores constantes. Es decir, es posible identificar el efecto del origen de los extranjeros en las variables respuestas (empleo o desempleo e inserción en ocupaciones descalificadas) en la medida en que las características de los trabajadores son controladas por los estimadores de las variables independientes insertadas en la regresión estadística.

En las dos ecuaciones el grupo de referencia son los inmigrantes brasileños, en la medida en que se pretende probar por medio de las estimaciones que ellos mantienen una distancia social mayor con los nativos (comparados con el resto de extranjeros latinoamericanos) y, por tanto, suelen estar en peor situación en el mercado laboral que los demás grupos de inmigrantes analizados, dado que tienen menos oportunidades de estar empleados en el mercado local y, cuando lo están, presentan más probabilidades de incorporarse al mismo con empleos de baja calificación. Complementariamente, también se pretende probar que, en comparación con los brasileños (el grupo menor en números absolutos entre los latinoamericanos), los ecuatorianos (el grupo mayor) se encuentran en peor situación

19 El sector flexible es la suma de las siguientes categorías: "Wholesale and retail trade", "Hotels and restaurants", "Transportation and communications", "Financial services and insurance", "Private household services", 'Other services", "Health and social work", "Education".

20 El sector público es la suma de las siguientes categorías: "Public administration and defense" y "Real estate and business services". 
ocupacional en el mercado de trabajo, teniendo más posibilidades de estar empleados en ocupaciones de baja calificación, ya que pueden ser vistos como un grupo que representa una amenaza étnica. Cabe destacar que para la segunda ecuación seleccionamos apenas los trabajadores empleados.

Ecuación 1:

$$
\log \left\lceil\frac{P}{1-P}\right\rceil=\beta_{0}+\beta_{1} X_{1}+\cdots+\beta_{k} X_{k}+\varepsilon
$$

Donde,

$\mathrm{P}=$ probabilidad de $\mathrm{Y}=1$ (del individuo estar empleado)

$1-\mathrm{P}=$ probabilidad de $\mathrm{Y}=0$ (del individuo estar desempleado)

$\beta_{0}=$ probabilidad de que se produzca el hecho cuando todas las $\mathrm{X}$ (variables independientes) sean igual a cero (intercepto)

$\beta_{1} X_{1}+\cdots+\beta_{k} X_{k}$ zfecto de las variables explicativas (edadcen tiempomigra soltero bolivia colombia ecuador perú venezuela educ1 educ2 educ3) sobre la probabilidad de que se produzca el hecho (inclinación).

$\varepsilon=$ error estoxcástico

Ecuación 2:

$$
\log \left\lceil\frac{P}{1-P}\right\rceil=\beta_{0}+\beta_{1} X_{1}+\cdots+\beta_{k} X_{k}+\varepsilon
$$

Donde,

$\mathrm{P}=$ probabilidad de $\mathrm{Y}=1$ (del individuo estar en empleo de baja calificación)

$1-\mathrm{P}=$ probabilidad de $\mathrm{Y}=0$ (del individuo no estar en empleo de baja calificación)

$\beta_{0}=$ probabilidad de que se produzca el hecho cuando todas las $X$ (variables independientes) sean igual a cero (intercepto)

$\beta_{1} X_{1}+\cdots+\beta_{k} X_{k \geq f}$ fecto de las variables explicativas (edadcen hrstrab tiempomigra soltero bolivia colombia ecuador perúvenezuela educ1 educ2 educ3 rígido flexible público voluntario autónomo) sobre la probabilidad de que se produzca el hecho (inclinación).

$\varepsilon=$ error estocástico 


\section{RESULTADOS}

Con el objetivo de comprobar qué inmigrantes están en peor o mejor situación en el mercado de trabajo, mostramos en la siguiente tabla, Tabla 2, un análisis no controlado de la proporción de inmigrantes empleados y de los trabajadores empleados en ocupaciones de baja calificación.

En cuanto a la proporción media de trabajadores ocupados se observa que el nivel de participación en el mercado de trabajo no varía, en gran medida (todos con porcentajes por encima de 79\%), entre los grupos de inmigrantes, comparados con los brasileños. La excepción es para los colombianos que tienen menos probabilidades estadísticamente significativas de estar empleados.

En relación con la inserción en trabajos de baja calificación se constata que entre los colombianos y los ecuatorianos existen una proporción mayor de inmigrantes en tales empleos. Cabe recordar que esos son los grupos de inmigrantes mayores comparados con los demás, representando el 30,6\% y el 53,10\%, respectivamente, corroborando la hipótesis elaborada de que cuanto mayor sea el grupo étnico menor es su realización en el mercado de trabajo debido a la existencia de amenaza étnica.

Tabla 2 - Análisis no controlado de las diferencias en la proporción de trabajadores ocupados y trabajadores empleados en ocupaciones de baja calificación (Test Z)

\begin{tabular}{|c|c|c|}
\hline Origen & $\begin{array}{c}\text { Proporción de inmigrantes } \\
\text { empleados }\end{array}$ & $\begin{array}{c}\text { Proporción de inmigrantes en empleos } \\
\text { de baja calificación }\end{array}$ \\
\hline Brasil & 0,86 & 0,17 \\
\hline Bolivia & 0,84 & 0,24 \\
\hline Colombia & $0,79 * *$ & $0,27 * *$ \\
\hline Ecuador & 0,87 & $0,37 * * *$ \\
\hline Perú & 0,84 & 0,23 \\
\hline Venezuela & 0,83 & $0,09 * *$ \\
\hline
\end{tabular}

Fuente: Censo demográfico español, 2001 (submuestra) - IPUMS. Datos trabajados por la autoras.**significativo al nivel $5 \% * * *$ significativo al nivel $1 \%$. 
Siempre con el objetivo de comprender de forma más detallada la inserción de los inmigrantes en el mercado de trabajo español, la tabla 3 muestra, a continuación, la distribución de los trabajadores por grupo ocupacional. Es de destacar que el censo demográfico de España, usado como fuente de datos para el análisis en este trabajo, agrupa las ocupaciones existentes en el mercado en 10 categorías con base en la International Standard Classification of Occupations (ISCO).

Se advierte la prevalencia de brasileños en ocupaciones caracterizadas como “Trabajadores cualificados y artesanos de las industrias manufactureras y la construcción”. En cambio, entre los colombianos y ecuatorianos se observa mayor concentración de inmigrantes en el mercado de "Ocupaciones elementales". Entre los peruanos y venezolanos hay mayor incidencia de inmigrantes como "Trabajadores de los servicios y vendedores de comercios y mercados".

En este sentido, a partir de tales datos hay indicios contrarios al discurso homogeneizador de la inserción de los inmigrantes en el mercado laboral español, muy presente en la bibliografía existente. Es decir, se sostiene que esos inmigrantes se incorporan mayoritariamente en los estratos más bajos y desvalorizados de la estructura ocupacional del mercado de trabajo español. No obstante, se observa una incidencia de inmigrantes inseridos en diversas ocupaciones (Ripoll, 2008).

Tabla 3 - Proporción de trabajadores por grupo ocupacional y por origen

\begin{tabular}{|l|c|c|c|c|c|c|}
\hline \multirow{2}{*}{ Grupo ocupacional } & \multicolumn{6}{c|}{ Origen } \\
\cline { 2 - 8 } & Bolivia & Brasil & Colombia & Ecuador & Perú & Venezuela \\
\hline Directores y gerentes & 3,65 & 8,18 & 4,48 & 2,22 & 5,99 & 15,38 \\
\hline $\begin{array}{l}\text { Técnicos y profesionales } \\
\text { científicos e intelectuales }\end{array}$ & 4,38 & 8,18 & 3,62 & 1,83 & 5,49 & 8,46 \\
\hline Técnicos; profesionales de apoyo & 5,11 & 9,09 & 5,21 & 3,25 & 5,99 & 15,38 \\
\hline $\begin{array}{l}\text { Personal de apoyo administrativo } \\
\text { Trabajadores de los servicios y } \\
\text { vendedores de comercios y } \\
\text { mercados }\end{array}$ & 3,65 & 6,36 & 3,4 & 2,68 & 3,99 & 5,38 \\
\hline
\end{tabular}




\begin{tabular}{|l|c|c|c|c|c|c|}
\hline $\begin{array}{l}\text { Trabajadores cualificados en el } \\
\text { sector agrícola, ganadero, forestal } \\
\text { y pesquero }\end{array}$ & 4,38 & 0,91 & 3,76 & 4,09 & 2,49 & 2,31 \\
\hline $\begin{array}{l}\text { Trabajadores cualificados y } \\
\text { artesanos de las industrias } \\
\text { manufactureras y la construcción }\end{array}$ & 27,01 & 26,36 & 27,0 & 26,61 & 22,94 & 18,46 \\
\hline $\begin{array}{l}\text { Operadores de instalaciones y } \\
\text { maquinaria, y montadores }\end{array}$ & 8,76 & 4,55 & 4,12 & 5,2 & 3,49 & 3,85 \\
\hline Ocupaciones elementales & 24,09 & 17,27 & 27,69 & 37,12 & 23,44 & 9,23 \\
\hline Ocupaciones militares & 0 & 0 & 0,22 & 0,04 & 0 & 0,77 \\
\hline \multicolumn{1}{|c|}{ Total } & $100 \%$ & $100 \%$ & $100 \%$ & $100 \%$ & $100 \%$ & $100 \%$ \\
\hline
\end{tabular}

Fuente: Censo demográfico español, 2001 (submuestra) - IPUMS. Datos trabajados por las autoras.

A continuación, las estimaciones realizadas por medio de regresiones logísticas, en las que la variable respuesta es 1 para individuos empleados y 0 para desempleados, indican que, en general, el origen de estos trabajadores latinoamericanos no aparece como una variable relevante para explicar la probabilidad de inserción del inmigrante en el mercado de trabajo español, tomando como referencia el grupo de brasileños. Los resultados señalan que, entre los inmigrantes analizados en este estudio, ningún grupo mostró coeficientes de regresión estadísticamente significativos $\mathrm{y}$, por tanto, existen las mismas oportunidades entre estos inmigrantes latinos con respecto a la posibilidad de estar empleados, al considerar el origen de los mismos.

Este resultado refuta la hipótesis, basada en la teoría de la distancia social, de que los brasileños, por haber sido colonizados por Portugal y no por España y debido a que el idioma oficial es el portugués y no el español, mantienen una distancia social mayor con los nativos, comparados con otros extranjeros latinoamericanos y, por tanto, suelen estar en peor situación en el mercado laboral que los demás inmigrantes provenientes también de América Latina. En cambio, se propone que los latinoamericanos en el mercado español son asimilados como grupos más homogéneos en cuanto a las posibilidades de encontrar empleo, de acuerdo con los estudios de Fernández y Ortega (2008), Amuedo-Dorantes y De la Rica (2006). 
Tabla 4 - Exponenciales de los estimadores $(\operatorname{EXP}(b))$ de las ecuaciones logísticas binomiales para el análisis de la empleabilidad

\begin{tabular}{|c|c|}
\hline Origen (Brasil como referencia) & $(\mathbf{E X P}(\mathbf{b}))^{*}$ \\
\hline Bolivia & 0,794 \\
\hline Colombia & 0,613 \\
\hline Ecuador & 0,981 \\
\hline Perú & 0,942 \\
\hline Venezuela & 0,825 \\
\hline
\end{tabular}

Fuente: Censo demográfico español, 2001 (submuestra) - IPUMS. Datos trabajados por las autoras.

* Para el cálculo de la probabilidadse ha empleado la fórmula siguiente: (EXP(b)-1)*100

En relación con las estimaciones realizadas por medio de regresión logística con la variable respuesta referida a la inserción en trabajos de baja calificación, los resultados muestran que con respecto a los inmigrantes de Ecuador el origen aparece como una variable estadísticamente significativa para el análisis de la posición ocupacional. Estos resultados sugieren que entre los grupos analizados en este trabajo, los ecuatorianos, comparados con los brasileños, tienen menos posibilidades ( $83 \%$ menos) de emplearse en puestos de baja calificación del mercado laboral español. Por tanto, a pesar de que se observa una mayor incidencia de trabajadores en este grupo ocupacional, el origen ecuatoriano reduce las probabilidades de que los inmigrantes se empleen en ocupaciones de baja calificación al compararlos con los brasileños. En este sentido, se refuta la hipótesis de que comparado con los brasileños (grupo menor en números absolutos entre los latinoamericanos), los ecuatorianos (el grupo mayor) se hallan en peor situación ya que pueden ser vistos como una amenaza étnica.

Este resultado arroja luz sobre los modelos de incorporación de los inmigrantes al mercado de trabajo basados en las redes sociales, que se centran en los efectos positivos de los grandes grupos de inmigrantes, como es el caso de los ecuatorianos. Estos investigadores argumentan que, debido a la conexión y a los lazos existentes entre los inmigrantes del mismo origen nacional, dichos trabajadores co-étnicos tienen un desempeño mejor en el mercado laboral (Portes y Bach, 1985; Portes y Rumbaut, 2001; Van Tubergen, Maas y Flap, 2004), dado su "capital étnico". El capital étnico, medido muchas veces por las redes sociales, la economía étnica y el enclave étnico, puede proporcionar a los inmigrantes empleos que no son accesibles en el mercado de trabajo abierto y accesible a los nativos y a otros inmigrantes. 
Tabla 5 - Exponenciales de los estimadores $(\operatorname{EXP}(b))$ de las ecuaciones logísticas binomiales para el análisis de la inserción en ocupaciones de baja calificación

\begin{tabular}{|c|c|}
\hline Origen (Brasil como referencia) & $(\mathbf{E X P}(\mathbf{b}))$ \\
\hline Bolivia & 1,205 \\
\hline Colombia & 1,542 \\
\hline Ecuador & $1,839^{* *}$ \\
\hline Perú & 1,630 \\
\hline Venezuela & 0,574 \\
\hline
\end{tabular}

Fuente: Censo demográfico español, 2001 (submuestra) - IPUMS. Datos trabajados por las autoras.

* Para el cálculo de la probabilidad se ha empleado la fórmula siguiente: $(\operatorname{EXP}(b)-1) * 100$

\section{CONSIDERACIONES FINALES}

Este artículo pretende verificar la inserción de los inmigrantes latinoamericanos, de origen brasileño, ecuatoriano, colombiano, venezolano, peruano y boliviano en el mercado laboral español. Para ello, se propone responder las siguientes cuestiones: ¿Existe desigualdad con respecto a la empleabilidad e inserción socio-ocupacional entre los trabajadores latinos en el mercado español? Si existen desigualdades, ¿quién está en condiciones de empleabilidad e inserción socio-ocupacional mejores o peores? En ese sentido, ¿el origen étnico/nacional del trabajador es un factor explicativo de tales desigualdades?

Para responder a las preguntas planteadas se ha utilizado un enfoque cuantitativo a través de la aplicación de modelos estadísticos con datos del censo demográfico de España de 2001, a partir del cual se establece una submuestra de hombres inmigrantes. Con respecto a los modelos de análisis, calculamos dos ecuaciones de regresión logística, para la constatación de las diferencias relativas al origen étnico/nacional para la determinación de la empleabilidad y para la inserción laboral de estos trabajadores. Para ello, en la primera estimación la variable respuesta es binaria, siendo que 1 significa que la persona está inserida en el mercado y 0 se refiere a las que están en paro y, para la segunda estimación, 1 significa que la persona está inserida en una ocupación de baja calificación y 0 se refiere a las que no están en ese tipo de puestos en el mercado laboral.

A partir de estos modelos ponemos a prueba dos hipótesis: 1) basándonos en la teoría de la distancia social, conjeturamos que los brasileños (por el hecho de que Brasil fue 
colonizado por Portugal y no por España y debido a que el idioma oficial es el portugués y no el español) mantienen una distancia social mayor con los nativos (comparados con el resto de extranjeros latinoamericanos). Por lo tanto, suelen estar en peor situación en el mercado de trabajo que otros inmigrantes también latinoamericanos; 2) basándonos en la teoría de la "amenaza étnica", sugerimos que, cuanto mayor es el grupo étnico, menor es la realización en el mercado laboral.

Por otro lado, siguiendo las teorías de la economía étnica (redes sociales y enclave étnico) conjeturamos que grupos grandes de inmigrantes tienen un desempeño mejor en el mercado de trabajo (PORTES Y BACH, 1985; PORTES Y RUMBAUT, 2001; VAN TUBERGEN, MAAS Y FLAP, 2004) debido a su capital "étnico". De esta forma, si refutamos nuestra segunda hipótesis mencionada anteriormente, estaremos corroborando las teorías de la economía étnica.

En cuanto a los resultados, en primer lugar, a partir de un análisis descriptivo de los datos, se constata que el nivel de participación en el mercado laboral no varía mucho, en gran medida (todos con porcentajes por encima de 79\%), entre los grupos de inmigrantes comparados con los brasileños. La excepción es para los colombianos que tienen menos probabilidades, estadísticamente significativas, de estar empleados.

En segundo lugar, las estimaciones realizadas a través de las regresiones logísticas, en las que la variable respuesta se refiere a 1 para individuos empleados y 0 para desempleados, señalan que, en general, el origen de estos trabajadores latinoamericanos no aparece como una variable relevante para explicar la probabilidad de inserción del inmigrante en el mercado de trabajo español, teniendo como referencia al grupo de brasileños. Este resultado refuta la hipótesis 1, basada en la teoría de la distancia social, de que los brasileños, por haber sido colonizados por Portugal y tener como lengua materna el portugués y no el español, mantienen una distancia social mayor con los nativos, comparados con otros extranjeros latinoamericanos y, por tanto, suelen estar en peor situación en el mercado de trabajo que otros inmigrantes provenientes también de América Latina. A partir de este resultado, podemos argumentar que, con respecto a la empleabilidad en el mercado español, estos grupos pueden ser analizados de manera conjunta de acuerdo con los estudios de Fernández y Ortega (2008), Amuedo-Dorantes y De la Rica (2006), dado que estos inmigrantes tienen las mismas posibilidades de estar empleados o no. 
Por último, respecto a las estimaciones realizadas por regresión logística con la variable respuesta referida a la inserción en trabajos con baja calificación, los resultados indican que sólo en el caso de los inmigrantes de Ecuador el origen aparece como una variable estadísticamente significativa para el análisis de la posición ocupacional. Se constata que el origen ecuatoriano reduce las probabilidades de que los inmigrantes se empleen en ocupaciones de baja calificación, tomando como grupo de referencia a los brasileños. En este sentido, se refuta la hipótesis de que, comparado con los brasileños (el grupo menor en números absolutos entre los latinoamericanos), los ecuatorianos (el grupo mayor) se encuentran en peor situación ya que pueden ser vistos como una amenaza étnica. Este resultado muestra la necesidad de comprensión del mercado laboral en España por medio de modelos de incorporación de los inmigrantes basado en redes sociales, que se centran en los efectos positivos de grupos grandes de inmigrantes. En este punto sugerimos que sean realizados estudios sobre el asunto futuramente.

En el caso del análisis de la posición en la estructura ocupacional del mercado de trabajo español, podemos inferir que no se deben analizar los grupos de manera agregada, como sucedió en estudios anteriores, expuestos anteriormente. La inserción de estos y otros grupos en el mercado laboral no se produce de manera homogénea y por lo tanto la misma debe ser analizada teniendo en cuenta las especificidades de cada grupo de origen étnico/nacional. 


\section{BIBLIOGRAFÍA}

AGUIAR, N. O. Desigualdades sociais, redes de sociabilidade e participação política. Belo Horizonte: UFMG, 2007.

AMUEDO-DORANTES, C.; DE LA RICA, S. The role of segregation and pay structure on the gender wage gap: evidence from matched employer-employee data for Spain. The BE Journal of Economic Analysis \& Policy, v. 5, n. 1, 2006. ISSN 1935-1682.

ARTILES, A. M.; LÓPEZ-ROLDÁN, P.; MOLINA, Ó. Movilidad ascendente de la inmigración en España:i asimilación o segmentación ocupacional? Papers: revista de sociologia, v. 96, n. 4, p. 1335-1362, 2011. ISSN 2013-9004.

BECKER, G. Investment in human capital: a theoretical analysis. Journal of Political Economy, v. 70, n. 5, p. 9-49, 1962.

BORJAS, G. J. Friends or strangers: the impact of immigrants on the US economy. New York: Basic Books, 1990.

Labor economics. New York: McGraw-Hill, 2005.

CHISWICK, B. R. Are immigrantes favorably self-selected? The American Economic Review, v. 89, n. 2, p. 181-185, 1999. Disponible en: < http://www.jstor.org/pss/117103 >.

COLEMAN, J. S. Social capital in the creation of human capital. American journal of sociology, v. 94, n. Supplement: Organizations and Institutions: Sociological and Economic Approaches to the Analysis of Social Structure, p. S95-S120, 1988. ISSN 0002-9602.

COLLINS, R. The Credential Society: An Historical Sociology of Education and Stratification New York: John Wiley, 1979.

ESCRIBANO, A. I.; DE LERA, D. L.; BUJÁN, R. M. Los preferidos del siglo XXI: la inmigración latinoamericana en España. La inmigración en España: contextos y alternativas, 2002, Granada. Laboratorio de Estudios Interculturales. p.237-249.

EXTRANJERÍA, D. D. G. P. L. I. Y. L. Balance 2003. Ministerio del interior. Madrid. 2004 FERNÁNDEZ, C.; ORTEGA, C. Labor market assimilation of immigrants in Spain: employment at the expense of bad job-matches? Spanish Economic Review, v. 10, n. 2, p. 83107, 2008. ISSN 1435-5469.

GIL ARAUJO, S. Inmigración latinoamericana a España: estado de la cuestión. Revista Gloobal, n. 5, 2005.

HASENBALG, C.; VALLE SILVA, N. D. Origens e destinos - desigualdades sociais ao longo da vida. Rio de Janeiro: Topbooks, 2003. ISBN:. Disponible en: < http://www.travessa.com.br/ORIGENS_E_DESTINOS_DESIGUALDADES_SOCIAIS_AO _LONGO_DA_VIDA/artigo/98a06b12-69d5-490a-9f5b-34e3e051942d >. 
IZQUIERDO, M.; LACUESTA, A.; VEGAS, R. Assimilation of immigrants in Spain: A longitudinal analysis. Labour Economics, v. 16, n. 6, p. 669-678, 2009. ISSN 0927-5371.

KESLER, C.; HOUT, M. Entrepreneurship and immigrant wages in US labor markets: A multilevel approach. Social Science Research, v. 39, n. 2, p. 187-201, Mar 2010. ISSN 0049-089X.

LIN, N. Social capital: a theory of social struture and action. Cambridge: Cambridge University Press, 2006.

MINCER, J. Investment in human capital and personal income distribution. Journal of Political Economy, v. 66, n. 4, p. .281-302, 1958.

NEE, V.; SANDERS, J. Understanding the diversity of immigrant incorporation: a forms-ofcapital model. Ethnic and Racial Studies, v. 24, n. 3, p. 386-411, May 2001. ISSN 0141-9870. Disponible en: <<Go to ISI >://000168803300002 >.

PICHLER, F. Success on European Labor Markets: A Cross-national Comparison of Attainment between Immigrant and Majority Populations1. International Migration Review, v. 45, n. 4, p. 938-978, 2011. ISSN 1747-7379.

PIORE, M. Birds of passage: Migrant labor and industrial societies. Cambridge University Press Cambridge, 1979.

PORTES, A. Capital social: origens e aplicações na Sociologia contemporânea. Sociologia, problemas e práticas, n. 33, p. 133-158, 2000.

PORTES, A.; BACH, R. Latin journey: Cuban and Mexican immigrants in the United States. Univ of California Pr on Demand, 1985.

PORTES, A.; RUMBAUT, R. G. Legacies : the story of the immigrant second generation. Berkeley, Calif. [u.a.]; New York, NY: University of California Press ; Russell Sage Foundation., 2001. ISBN 0520228472978052022847405202284809780520228481.

PORTES, A.; SHAFER, S. Revisiting the enclave hypothesis: Miami twenty-five years later. Research in the Sociology of Organizations, v. 25, p. 157-190, 2007.

RIPOLL, E. M. O Brasil e a Espanha na dinâmica das migrações internacionais: um breve panorama da situação dos emigrantes brasileiros na Espanha. Revista Brasileira de Estudos de População, v. 25, n. 1, p. 151-65, 2008.

RODRÍGUEZ-PlanAS, N.; NOLLENBERGER, N. A Precarious Position: The Labor Market Integration of New Immigrants in Spain. Washington: Migration Politicy institute, 2014.

SANROMÁ, E.; RAMOS, R.; SIMÓN, H. Immigrant wages in the Spanish labour market: does the origin of human capital matter? IZA discussion papers, n. 4157, 2009. 
SASSEN KOOB, S. The mobility of Labor and Capital: A study in international investment and labor flow. Cambridge: Cambridge University Press, 1988.

TIENDA, M.; LII, D. T. Minority concentration and earnings inequality - blacks, hispanics, and asians compared. American Journal of Sociology, v. 93, n. 1, p. 141-165, 1987. ISSN 00029602.

VAN TUBERGEN, F.; MAAS, I.; FLAP, H. The economic incorporation of immigrants in 18 western societies:origin, destination, and community effects. American Sociological Review, v. 69, n. 5, p. 704-727, 2004. Disponible en: < http://www.jstor.org/stable/3593035 >.

VILELA, E. M. Desigualdade e discriminação de imigrantes internacionais no mercado de trabalho brasileiro. Dados - Revista de Ciências Sociais, v. 54, n. 1, p. 89-129, 2011.

VILELA, E. M.; COLLARES, A. C. M.; NORONHA, C. L. A. D. Migrações e trabalho no Brasil: fatores étnico-nacionais e raciais. Revista Brasileira de Ciências Sociais, v. 30, n. 87, p. 19-42, 2015. ISSN 0102-6909.

WILSON, K. L.; PORTES, A. Immigrant enclaves: An analysis of the labor market experiences of Cubans in Miami. American journal of Sociology, p. 295-319, 1980. ISSN 0002-9602.

Recebido em 27/02/2018. Aceito em 27/04/2018. Publicado em 28/08/2018. 